\title{
The Prospective Cognition of Food Caching and Recovery by Western Scrub-Jays (Aphelocoma californica)
}

\author{
Nicola S. Clayton, Nathan J. Emery and Anthony Dickinson \\ University of Cambridge
}

\begin{abstract}
The role of prospective cognition in food caching and recovery by western scrub-jays (Aphelocoma californica) is reviewed. These birds anticipate the short-term consequences of searching for cached food at recovery by reducing their searches for devalued food items. Two further lines of evidence suggest that the jays are also capable of more long-term prospection. First, the caching of food items decreases when they are consistently degraded or pilfered at recovery over cache-recovery intervals that preclude direct delayed reinforcement and punishment. Second, the jays anticipate the pilfering of their caches by another bird, which observes the caching episode, by engaging in various cache-protection behaviors. These finding suggest that the jays are capable of a form of prospective mental "time travel".
\end{abstract}

The last decade has seen an increasing interest in prospective and retrospective cognition by non-human animals. In part, this interest has its origin in Tulving's (1983) claim that only humans have an episodic memory system, which allows them to mentally travel through subjective time to reminisce about specific past events. While animals can acquire extensive general information about their environment, according to Tulving $(1983$, p. 1) "they cannot travel back in the past in their own minds". The scope of this "temporal myopia" was extended from the past to future by Suddendorf and Corballis (1997) in the formulation of their 'mental time travel hypothesis', which claims that animals live in the present, being incapable of episodic recall of specific past events and unable to contemplate possible states of affairs beyond the immediate future. Suddendorf and Corballis based their hypothesis on a review of primate cognition. More recently, Roberts (2002) reached a similar conclusion that animals are, to use his words, "stuck in time" by reThe research reviewed in this article and its preparation was supported by grants from the Biotechnology and Biological Science Research Council (S16565 and BBS/B/053434) and was conducted within the Medical Research Council Co-operative Grant G9805862. Nathan Emery is supported by a Royal Society University Research Fellowship. The photograph was taken by Nicky Clayton and Ian Cannell, University of Cambridge.

Correspondence concerning the article should be addressed to Professor Nicola S. Clayton, Department of Experimental Psychology, University of Cambridge, Downing Street, Cambridge CB2 3EB, United Kingdom. E-mail: nsc22@cam.ac.uk

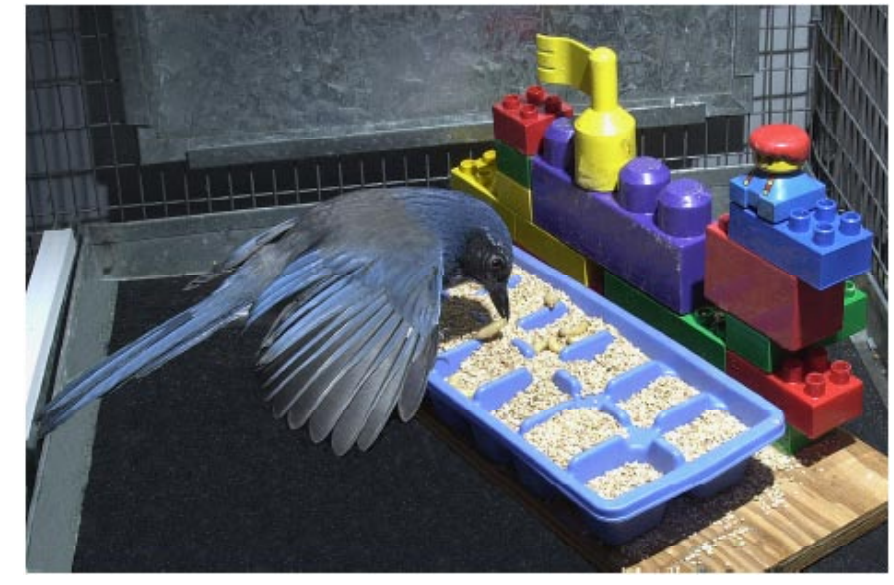

Figure 1. Western Scrub-Jay (Aphelocoma californica) caching food.

viewing the evidence from studies of a variety of species. Moreover, in recent writings, Tulving has also endorsed the prospective component of the mental time travel hypothesis by stating that "mental time travel allows one, as an "owner" of episodic memory ("self"), through the medium of autonoetic awareness, to remember one's own previous "thoughtabout" experiences, as well as to "think about" one's own possible future experiences" (Tulving, 2005, pp. 9).

About 10 years ago, we started a research program on the retrospective component of the mental time travel hypothesis in which we investigated the mnemonic processes mediating the recovery of food caches by western scrub-jays. Many species scatter-hoard food throughout their territories when 
it is abundant, only to recover it at some later time of scarcity (Vander Wall, 1990), and it has long been known that recovery is based, at least in part, on memory for the location of the caches. What was unknown at that time, however, was whether cachers remember not only where their caches are located but also what type of food was cached and when the caches were made. There were good reasons to believe that scrub-jays might well encode such what-where-when memories. Unlike many specialist cachers, such as Clark's nutcrackers and pinion jays that are heavily dependent on the harvesting of pinyon seeds, scrub-jays cache a variety of food types, which include not only nuts and seeds but also perishable food items, such as invertebrates (Curry, Peterson \& Langen, 2002). Moreover, as these jays inhabit the central valley of California, in which the rate at which perishable caches degrade varies with changes in temperature from below $10^{\circ} \mathrm{C}$ to over $40^{\circ} \mathrm{C}$, there were good a priori, ecological reasons for believing that the jays might encode what-where-when information in their memory of caching episodes in order to be able to recover perishable caches before they degrade. And, indeed, we found good evidence for such encoding in a flexible, declarative form, which led us to attribute the capacity for episodic-like memory to the jays (see de Kort, Dickinson, \& Clayton, 2005, for a recent review).

Although cache recovery depends upon retrospective cognition, food-caching is a behavior that is oriented towards future needs. Indeed, the act of hiding food is without obvious immediate benefit, yielding its return when the bird comes to recover its caches days, if not weeks, later. A Clark's nutcracker, for example, may cache food in October and recover it up to nine months later. But like any other apparently prospective behavior, food-caching would not be an example of future planning if the animals were insensitive to the consequences of their actions. It is therefore important to distinguish mental time travel into the future from simple prospective behavior, because species-specific behaviors that appear to involve the anticipation of future states need not involve any planning ability. Indeed, ever since Fabre's (1916) classic observation that even minor perturbations in the stimulus configuration during nest provisioning by solitary wasps disrupted the whole complex behavioral sequence, the dangers of attributing intentionality to an animal on the basis of the manifest goal-directedness of its behavior, however complex and sophisticated, have been clear. It is now well established that the wasp's nest provisioning behavior is an innately determined fixed action pattern (Baerends, 1941). Many other prospectively-oriented behaviors may also be innate, such as the migratory orientation of black-capped warblers. For example, some German populations of black-capped warblers migrate southwest towards Africa in the winter, whereas others migrate west to Britain. When birds from these different populations were housed in captivity, and allowed to breed, the offspring showed the same migratory orientation as their genetic parents, irrespective of the environment in which they had been raised (Berthold, Heilbig, Mohr, \& Querner, 1992). So although this migratory behavior might appear to have some of the features of prospective behavior, it does not involve future planning.

Other apparently prospective behaviors may be no more than simple, reinforced habits. There are few of us who have not experienced slips of action caused by well-practiced behavior that are not appropriate to our current goals. These behaviors, although acquired through learning, are simply elicited by environmental stimuli. The famous example is that related by William James (1890) who, when going up to his bedroom to change for dinner, suddenly found he had put on his night gown and got into bed.

We think that scrub jays certainly have an innate motivation to cache. The fact that, in absence of a suitable food, they cache inedible objects, such as stones (Clayton \& Dickinson, 1999a), which are of no obvious future benefit, illustrates its compulsive nature. Moreover, caching is under local motivational control. Pre-feeding the jays a particular food, ether peanuts or dog kibbles, in a powdered, non-cacheable form not only produced a general reduction in subsequent caching, but also a more specific reduction in caching the prefed food type (Clayton \& Dickinson, 1999a). In this respect, caching exactly parallels feeding, suggesting that it is under the control of the current incentive value of the food. At issue, therefore, is whether this propensity is also modulated by the consequences of caching.

In this review, we shall begin by describing our general methods for studying caching and recovery by the jays before discussing whether searching for caches is under the control of short-term prospective cognition. We shall then consider whether caching itself is sensitive to its more long-term consequences. Two lines of evidence are brought to bear on this issue. The first is whether the degradation and pilfering of particular food caches before recovery selectively affect the subsequent caching of this type of food, whereas the second concerns the behavioral strategies that the jays employ to prevent the pilfering of their caches by conspecifics that might know where the food has been cached. Throughout the review, we shall be particularly concerned with the extent to which the effect of these variables is mediated by basic non-cognitive, reinforcement processes rather than by representations of the future consequences of caching and recovery.

\section{General Methods}

All the studies were conducted on a mixed-sex colony of hand-raised, sexually mature western scrub-jays. Although each bird has been studied in multiple experiments, we keep a complete record of its experimental history to ensure that 
its past experience did not confound the interpretation of subsequent results. Each bird was tested individually in its home cage and each trial consisted of at least 2 phases, one or more caching episodes followed by a recovery episode, separated by a retention interval. Caching took place in the morning and recovery in the afternoon. During the experiments the birds were maintained on powder food to prevent extra-experimental caches, which was removed just prior to the dark phases of the diurnal cycle before caching and $4 \mathrm{hr}$ before a recovery. Consequently, the jays were hungry at the time of both caching and recovery.

At the start of the caching phase, one or two caching trays were placed in the bird's cage along with a bowl containing the food items for caching. Each caching tray consisted of an ice-cube tray, which was surrounded by a structure of children's Lego Duplo® building bricks that rendered the tray trial-unique (see Figure 1). The individual moulds in the trays were filled with a caching substrate, sand or corn kibbles, to provide distinguishable sites in which the birds could cache the food. The jays had to use the structure of a particular tray to remember where in the tray different food items were hidden on a given trial. In addition, we could control the sites available for caching by placing a Perspex strip over one row of the tray moulds.

The video shows a typical caching episode in which there are two trays in the cage and a bowl of wax moth larvae (wax worms), the jay's favorite food. At the start of the video, the bird is out of view on a perch above the top of the screen. It then flies down to the floor and proceeds to destroy the surrounding structure of one of the trays by pecking off a brick before collecting a beak full of worms and depositing them on the floor. The first caching event takes place outside the trays. The bird takes a worm and caches out of view in the upper left-hand corner of the cage. Although we have designed the cages and floors to minimize potential cache sites, the jays often find places to cache within the cage itself. To discourage the birds from caching in locations other than those in the trays we provided ('illegal sites'), we attempt to remove all such caches following each caching episode. The jay then collects the remaining wax worms and proceeds to cache then in one of the moulds of the left-hand tray. Three features of this caching behavior are noteworthy. First, the jay deftly kills or stuns the worms before caching them so that they will not escape from the cache site. Second, having buried the worms in one of the moulds, the bird carefully covers up the cache with substrate from an adjacent mould. Finally, having completed the cache, the jay spends a few moments inspecting the surrounding structure with all the appearance of actively encoding the location of the cache. This behavior illustrates one of the advantages of the cacherecovery procedure - the jays do not have to be trained to cache and recover nor, apparently, to encode the relevant in- formation for successful recovery. The video ends with the bird collecting some more worms from the bowl, which it proceeds to cache in the right-hand tray.

At the end of the caching episode, we remove the trays from the home cage and note the location and types of food items cached before returning the trays to the cages for the recovery phase after the required retention interval. The conditions at recovery depend upon the purpose of the experiment. To study whether the birds use memory when searching at recovery, we remove or pilfer all the caches from the trays prior to returning them for the recovery phase. This pilfering ensures that no cues emanate from the caches themselves so that searching for the caches at recovery must be based on the jay's memory of the caching episode. We also pilfer some of the caches prior to recovery in studies of the state of the caches at recovery on caching itself. Alternatively, in other conditions, we artificially degrade certain cached food types by soaking them in detergent and green-colored quinine to render them unpalatable before returning them to the tray for recovery. Finally, as a control for these degrade and pilfer conditions, in the replenish condition we simply place fresh food items in the trays prior to recovery.

We should note the artificial nature of our generic cacherecovery procedure. In the wild, the jays leave and return to the caches sites at will, and thus the birds control the length of the retention interval. In our procedure, by contrast, a human experimenter removes the cache sites and returns them to the bird. Importantly, however, the jays' lack of control over the retention does not seem to disrupt their propensity to cache and recover.

\section{Short-Term Prospection}

There is good evidence to suggest that certain animals, at least, anticipate the immediate consequences of their actions. For example, Adams and Dickinson (1981) trained rats to lever press for one type of food, either mixed composition food pellets or sugar pellets, while presenting the other food when the rats were not pressing. At issue was whether the rats pressed the lever because they anticipated that this action would yield a particular type of pellet or, in other words, whether their behavior was controlled by short-term prospection. To address this question, following this leverpress training, Adams and Dickinson devalued one of the pellet types, either the contingent one that was caused by the lever press during training or the other, non-contingent type. The devaluation involved conditioning a food aversion to the respective pellet type in the absence of the opportunity to press the lever. Finally, to investigate whether this devaluation would impact on lever pressing, the rats once again had the opportunity to perform this action. Importantly, this test was conducted in the absence of either type of food pellet so that any change in performance must have been mediated by the rats' knowledge of the relationship between the 
lever pressing and the contingent pellet type acquired during initial training. As a consequence, lever pressing during the test should have reflected anticipation that this action would yield that pellet type during testing. Evidence that the rats did in fact anticipate the consequences of their action came from the fact they pressed least when the contingent pellets were devalued.

We used this basic devaluation procedure to investigate whether scrub-jays also anticipate the consequences of their searches during recovery (Clayton \& Dickinson, 1999b), with searching for caches at recovery playing the role of lever pressing and the cached foods the role of the reward pellets. The birds were initially allowed to cache peanuts in one tray and dog kibbles in another tray in separate caching episodes. Both trays were then returned to the jays' cages for the recovery phase during which the birds were free to search in either tray. This recovery phase took place in the afternoon of either the same day as caching or a week later. In fact, the birds could not recover any caches because we suggesting that it reduces the incentive value of the food. Therefore, if the jays anticipated the consequences of their searches at recovery, they should have searched more in the tray in which they had cached the non pre-fed food than in the one in which they had cached the pre-fed food. This is exactly the pattern observed during recovery.

We must be cautious, however, before accepting that this devaluation effect demonstrates that the jays anticipate the consequences of searching for their caches for reasons illustrated by a study of (Russell \& Thompson, 2003). They allowed children to observe the experimenter placing 2 attractive toys in separate boxes, before another experimenter entered the room and removed the toy from one of the boxes. The child was then asked to select one of the two boxes. Surprisingly, children below 20 months of age predominantly chose the box from which the toy had been recovered rather than the box that still contained the toy, and it was not until the children were about 2-yr old that they consistently selected this latter box. One account of the paradoxical prefer-

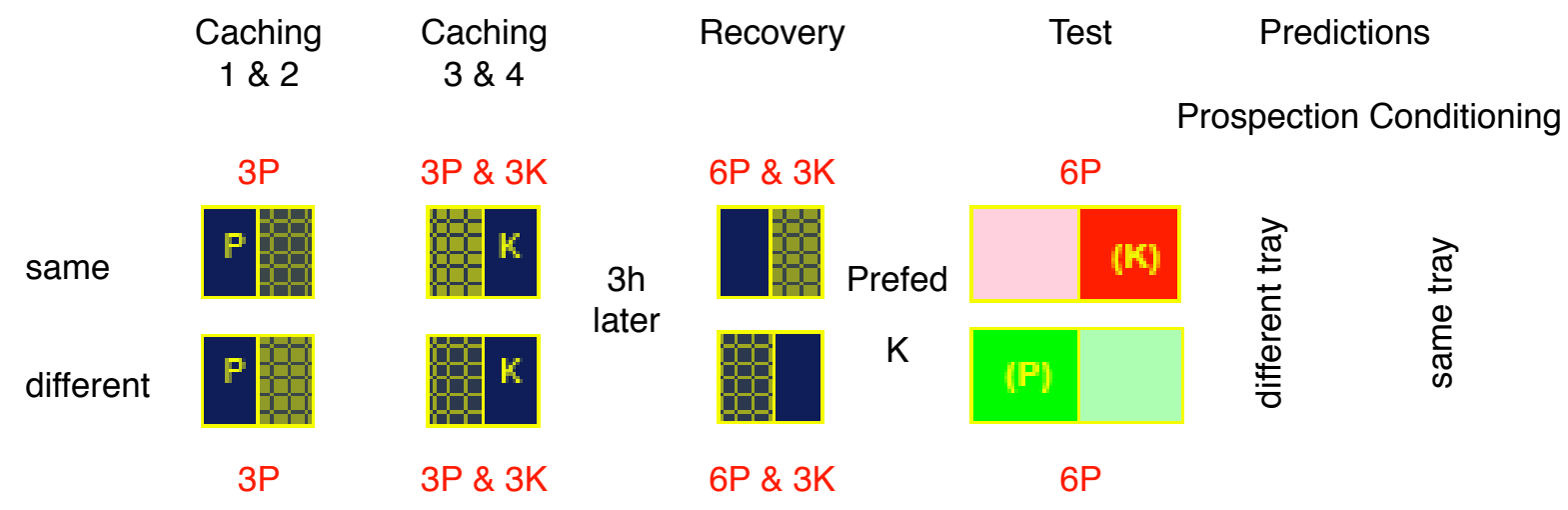

Figure 2. The design of Experiment 2 from Clayton and Dickinson (1999b) in which jays cached peanuts (P) and kibbles $(K)$ in different sides of the same and different trays. The yellow hatched areas represent the sides of the trays covered to prevent access. During the test the letters in parentheses indicate the foods that should have still been in the trays but were in fact removed by us before the test. Also shown are the predicted search preferences of the jays during the test predicted from accounts in terms of prospective cognition and Pavlovian conditioning (see text for details). The effective strength of the associations between each tray and the peanuts and kibbles are shown in red on the assumption that each pairing of a tray with a particular food items produces an increment of one in the corresponding associative strength. The color and shade of the sides of the trays on test corresponds to the types of searches shown in Figure 3.

had pilfered them prior to the recovery for the same reason that Adams and Dickinson (1981) did not present their rats with the food pellets during the test - to ensure that searching was based of the jay's memory of the caches.

In order to devalue one of the cached food types, immediately prior to the recovery phase we pre-fed the jays one of the foods in a powdered form, either the peanuts or the kibble in a counterbalanced design. We have already noted that such pre-feeding selectively reduces eating and caching of the pre-fed food (Clayton \& Dickinson, 1999a), strongly ence of the younger children notes that the empty box had received two pairings with toy, one during the initial baiting and the other during the recovery of the toy, whereas the box still containing the toy received only the single pairing during baiting of the boxes. Consequently, the greater number of pairings of the empty box with the toy may have conditioned a stronger preference for the younger children, who did not reason about the consequences of the experimenter's actions for the anticipated outcome of their own choices.

At issue, therefore, is whether the choices of scrub jays 
are similar those of younger children, reflecting a simple conditioned preference, or whether birds manifest a form of prospective cognition that corresponds to the apparent reasoning of older children. In fact, the Russell and Thompson (2003) study employed a simplified version of a design that we had used to address this issue with the jays (Clayton \& Dickinson, 1999b). Figure 2 illustrates this design. In the first of 2 caching episodes in the morning, the jays were allowed to cache 3 peanuts in one side of each of 2 trays; the same and different trays. Let us assume that the caching of each peanut produced an increment in the association between the tray and peanuts of $\mathrm{P}$ so that by the end of these caching episodes each tray should have an associative strength for peanuts of 3P (see Figure 2). Then, in the third and fourth caching episodes, the jays cached 3 dog kibbles in the other side of each tray, thereby establishing an associative strength of $3 \mathrm{~K}$ with the kibbles for both trays. Each tray was then returned to the birds $3 \mathrm{hr}$ later in the afternoon for the recovery episode during which they were allowed to recover the 3 peanuts from the same tray and the 3 kibbles from the different tray. According to the conditioning account, this recovery should have enhanced the associative strength of the same tray with peanuts to $6 \mathrm{P}$ and that of the different tray with kibbles to $6 \mathrm{~K}$. Remember that, as in the case of the younger children, Pavlovian conditioning is not sensitive to the type of interaction with the caches but simply to the pairings of the tray cues with the foods. Consequently, after the caching and recovery episodes, the same tray should have had associative strengths of $6 \mathrm{P}$ and $3 \mathrm{~K}$ with the peanuts and kibbles, respectively, whereas the corresponding associative strengths of the different tray would have been $3 \mathrm{P}$ and $6 \mathrm{~K}$. In terms of the remaining caches, however, the same tray contained only kibbles and the different tray only peanuts.

Following the caching and recovery episodes, we once again removed the trays from the home cages and then devalued one of the foods by pre-feeding it to the jays in a powdered form. Figure 2 shows the design in which the kibbles were pre-fed but in fact the type of pre-fed food was counterbalanced across the birds. It was the type of prefeeding that defined the designation of the trays. The same tray was the one that still contained caches of the same type, kibbles in this example, as the pre-fed food, whereas the different tray still contained the non-pre-fed food, in this case peanuts. Our assumption was that pre-feeding a food would render its associative strength with the tray cues ineffective in controlling the jays' tray preference in the subsequent test when they were given a choice between searching in the two trays. Consequently, in the current example, pre-feeding kibbles should have reduced the influence of any association between the trays and the kibbles on searching. Therefore, as a result of pre-feeding, the same tray should have had an effective associative value of $6 \mathrm{P}$ and the different tray a strength of only $3 \mathrm{P}$ so that the conditioning account predicts that the birds should have searched preferentially in the same tray during the test.

Figure 3 shows that in fact the jays' search preference on test was exactly the opposite of that predicted by the conditioning account; they showed more searching in the different tray than in the same one. Indeed, not only did they show a tray preference at variance with a conditioning explanation, they also searched selectively in the intact sides of the trays, which should have still contained caches, rather than in the sides from which they had previously recovery their caches.

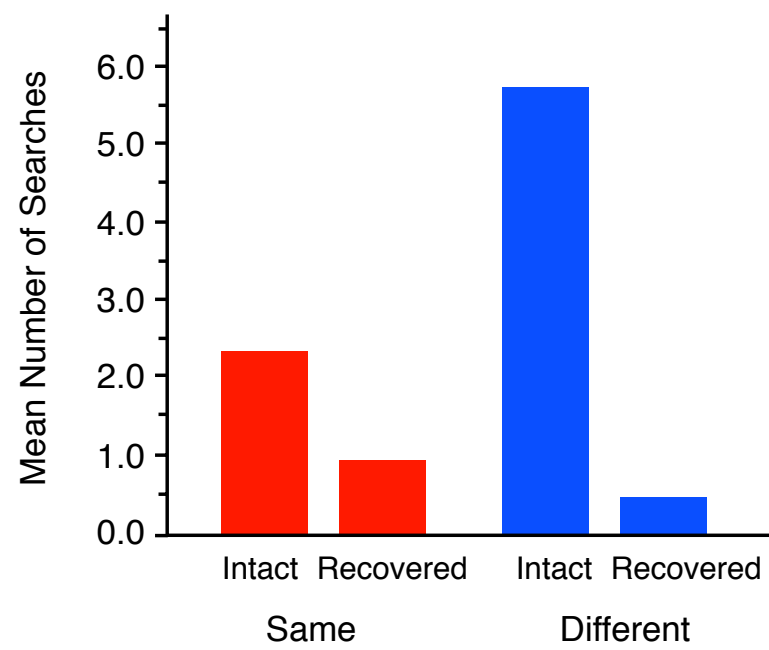

Figure 3. The mean numbers of searches directed by the jays to the intact and recovered sides of the same and different trays during the test of Experiment 2 of Clayton and Dickinson (1999b)

In fact, the pattern of searching on test is exactly that predicted by an account in terms of prospective cognition. As a result of their caching and recovery experiences, the jays should have anticipated that searching the intact sides of the trays would have yielded kibbles in the case of the same tray but peanuts in the case of the different one (see Figure 2), which explains the observed preference for searching the intact sides. The fact that this preference was much greater in the different tray than in the same tray reflects the fact that the caches that the birds expected to find in the different tray should have had a higher incentive value than those anticipated in the same tray following prefeeding.

In summary, jays anticipated the immediate consequences of their searches at recovery, a form of prospective cognition that cannot be easily explained in terms of the basic associative processes of Pavlovian conditioning. Whether or not other demonstrations of prospection in animals are equally problematic for an associative analysis is less clear. Many discriminative phenomena show behavioral control by outcome expectancies: acquired equivalence and distinctiveness, the differential outcomes effect, outcome-specific 
Pavlovian-instrumental transfer, to name but a few (Hall, 1996; Zentall, 1998). Although these forms of expectancymediated discrimination have been characterized as involving prospective processing (Honig \& Thompson, 1982), they are open to an analysis in terms of associations between the discriminative stimuli and outcomes. Perhaps more problematic for an associative account is demonstration that rats appears to switch from remembering the visited arms to remembering the yet-be-visited arms during the course of a trial in the radial-arm maze (Cook, Brown, \& Riley, 1985).

Whatever the involvement of non-associative prospective cognition in these tasks, we very much doubt that Suddendorf and Corballis, 1997, would accept these forms of short-term prospection as examples of the mental time travel. In fact, they formulate the prospective component of their hypothesis so that it specifically precludes short-term prospection by appealing to the writings of Wolfgang Köhler, Norbert Bischof, and Doris Bischof-Köhler, which they synthesize into what they call the Bischof-Köhler hypothesis. This hypothesis claims that "animals other than humans cannot anticipate future needs or drive states, and are therefore bound to a present that is defined by their current motivational state" (Suddendorf \& Corballis,1997). However, as we noted in the introduction, caching appears to be a behavior at variance with this claim - it is an action undertaken in the present with the apparent purpose of fulfilling a future need. The question is, however, what type of 'purpose' is it: Is it a psychological purpose reflecting the cacher's anticipation of a future need, or is it an illusionary teleology conferred by those two great selectors of behavior: natural selection on inclusive fitness and selection by reinforcement? In other words, if caching is either an innate behavioral propensity that is unmodulated by its consequences at recovery or a response that is directly reinforced by these consequences, there would be no need to appeal to any role for prospective cognition.

\section{Long-Term Prospection}

Our approach to this question has been two-fold. First, we have asked whether caching is sensitive to whether or not it fulfills a future need, presumably hunger, at recovery. If caching is insensitive to this long-term goal, it would seem very unlikely that it involves any form of prospective cognition, and so our first step was to establish whether or not caching is sensitive to its consequences at recovery. The second line of research arose from the observation that scrub-jays steal each others caches when given the opportunity to do so. As human mental time travelers, we ourselves go to great length to protect our valued possessions against future theft, which raises the question of whether jays also attempt to protect their caches against the potential thief.

\section{The Consequences of Caching}

It is has long been known that food-caching birds avoid caching in sites that are consistently pilfered relative to those that remain intact (Hampton \& Sherry, 1992; Kamil, Balda, Olson, \& Good, 1993). However, this preference can simply be explained in terms of a conditioned preference for the intact sites reinforced by food at the time of recovery and the absence of such reinforcement for the pilfered sites. Less readily explained in terms of conditioning at the time of recovery would be a reluctance to cache a particular food type

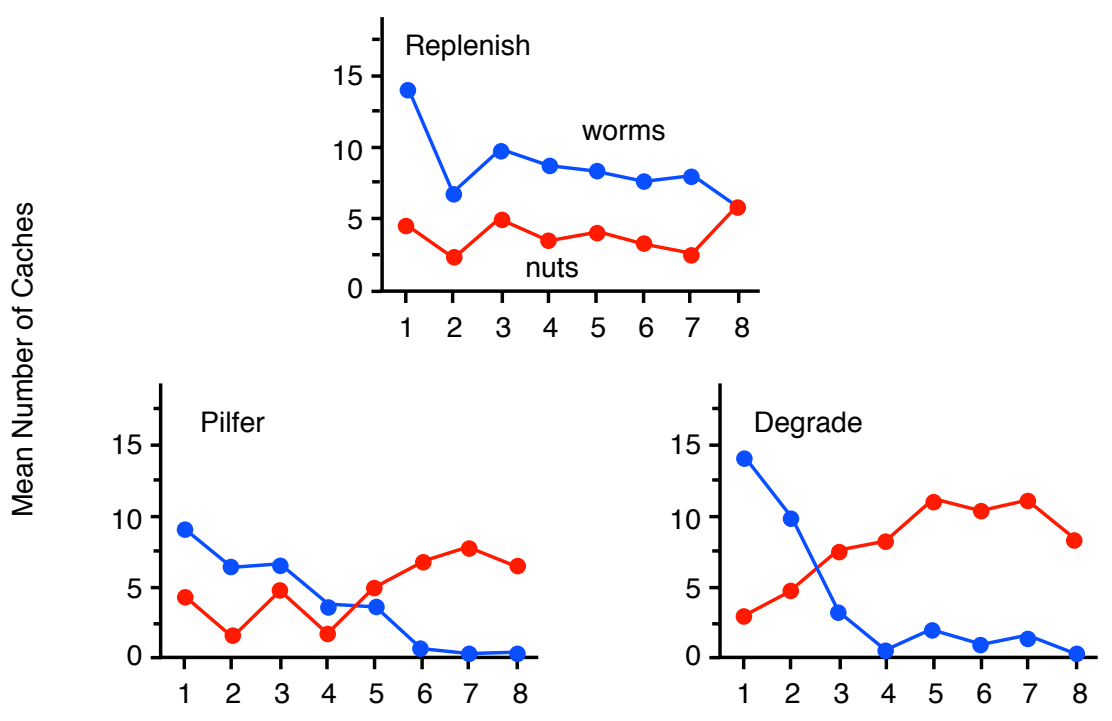

Trial

Figure 4. The number of wax worms and peanuts cached by groups of scrub-jays when wax worms caches were replenished with fresh worms prior to recovery, pilfered, or degraded in Experiment 2 of Clayton et al. (2005). 
that is consistently pilfered, and therefore, along with our colleagues, we investigated whether the consistent pilfering of a particular food would affect the caching of that food (Clayton, Dally, Gilbert, \& Dickinson, 2005).

On each trial, the jays were free to cache as many wax worms and peanuts as they liked in the tray for $15 \mathrm{~min}$ before it was removed. The tray was returned for recovery in the afternoon of the same day on half of the trials but 4 days later on the remaining trials. The peanut caches were always intact at recovery. As the top panel of Figure 3 illustrates, if the wax worm caches were replenished with fresh worms prior to each recovery, the birds showed a sustain propensity (except on the last trial) to cache more wax worms than peanuts, presumably reflecting their preference for wax worms over peanuts. By contrast, when we consistently pilfered the wax worms caches prior to recovery, the jays progressively cached fewer worms, but more peanuts until after 5 trials they had almost ceased caching any worms. Clearly, the jays were sensitive to the consequences of caching.

Although this pilferage condition produced relatively rapid learning, the failure to find a cache at recovery provides ambiguous information in the sense that the jay may fail to find the cache either because it has been pilfered or because the bird has forgotten the location of the cache. Consequently, we also tested another group of jays in a third, degrade condition in which we restocked the worm caches with artificially degraded, unpalatable worms, which the jays spat out when recovered. A comparison of the bottom panels of Figure 4 shows that the jays learned to decrease caching worms and increase caching peanuts in this degrade condition even more rapidly than in the pilfer condition. After just 2 trials, the jays were caching less wax worms than peanuts and by the fourth trial they had almost given up caching wax worms at all. This reduction in caching was not due to a change in the attractiveness of fresh wax worms because the birds in the degrade group showed just as strong a consumption preference for worms over peanuts as those in the replenish group. That is, when given a choice between eating the two types following the caching training, the degrade group showed an almost exclusive preference for eating the fresh worms.

At the very least, these results demonstrate that scrub-jays are sensitive to the consequences of caching and, in this respect, differ from some other food-storing animals. (McKenzie, Bird, \& Roberts, 2005) have recently reported that rats, unlike the jays, continue to cache in locations in which the food is degraded or from which it is pilfered even with a retention interval of only $45 \mathrm{~min}$. At most, the sensitivity of the jays to the consequences of caching challenges the prospective component of the mental time travel hypothesis by demonstrating that the birds are sensitive to the relevance of their caches to the state of hunger at the time of recovery.
A decrement in the hunger-relevant incentive value of the caches, either by pilferage or degradation, reduced caching.

Before endorsing this conclusion, however, we should consider alternative explanations that do not invoke longterm prospective cognition. It seems unlikely to us that caching of the worms was maintained by the direct, delayed reinforcing effect of recovering the worms on caching. Such an account would assume that the decline in worm caching reflects simple extinction in the pilfer condition and possible even delayed punishment in the degrade condition. The reason for our skepticism is that the reinforcement and punishment would have to have operated across delays, $4 \mathrm{hr}$ on half of the trials and $100 \mathrm{hr}$ on the remaining trials, which are orders of magnitude longer than other demonstrations of delayed positive reinforcement and punishment. However, to check that caching is sensitive to its consequences across a delay that renders the reinforcement account implausible, we replicated the degrade condition with a consistent retention interval of 2 days (Clayton et al., 2005). Again the jays reduced their caching of worms to well below that of peanuts after only 2 trials.

It remains possible, however, that caching is controlled by some form of mnemonically mediated reinforcement and punishment process. Our studies of retrospective cognition in the cache-recovery paradigm have established that jays remember the caching episode at recovery. Therefore, the jays may recall the act of caching worms during recovery, which then allows this behavior to be indirectly reinforced, punished, or extinguished by the fresh, degraded and pilfered worms, respectively. This possibility must be taken seriously in view of the extensive evidence for mnemonic mediation (Holland, 1990). An alternative explanation is that at the time of caching, the jays remember the state of worm caches during previous recovery episodes and use this information to decide whether or not to cache the worms, a process that represents a form of prospective cognition. At present, our data cannot distinguish between these alternatives.

\section{Cache Protection}

As alluded to in the introduction to this section on longterm prospection, other individuals, so-called pilferers, may steal caches. Such theft is particularly problematic for scrubjays and other members of the corvid family, where potentially pilfering conspecifics use observational spatial memory to accurately steal another's caches that they saw being made. Pilfering jays can wait until the cacher has left the scene and then steal its caches at will, whenever they are hungry, and without relying on successfully displacing a possibly more dominant cacher. Bugnyar and Kotrschal (2002) suggested that the capacity for observational spatial memory in corvids provides the catalyst for an 'evolutionary arms race' between cachers and pilferers, such that pilferers develop methods 
for observing the cachers as unobtrusively as possible, and cachers develop strategies to counter the risk of cache pilferage. Furthermore, Dally, Emery and Clayton (in press a) have argued that, because each jay can act as both cacher and pilferer, this dual role leads to a refinement of increasingly more sophisticated, cognitively-based cache protection and pilfering strategies. So, surely, if scrub-jays are capable of prospective cognition, then a jay who has cached food when other individuals were present should take protective action to minimize the likelihood that those other individuals could steal the caches at a later date.

So far our studies have focused on the behavior of the cachers, notably their ability to anticipate pilferage and the strategies they use to protect their caches from being stolen by other birds. Field observations suggest that cachers engage in a number of cache protection strategies, such as waiting until would-be pilferers are distracted or cannot see before caching (e.g. ravens, Corvus corax: Heinrich, 1999; Heinrich \& Pepper 1998), or by caching in areas of low conspecific density (e.g. rooks, Corvus frugilegus: Kalländer 1978; magpies, Pica pica: Clarkson, Eden, Sutherland, \& Houston, 1986; ravens: Bugnyar \& Kotrschal 2002). Some corvid species are also known to return alone to caches hidden in the presence of conspecifics, and move them to new locations unbeknown to potential pilferers (e.g. ravens: Heinrich, 1999; Eurasian jays, Garrallus glandarius: Goodwin, 1955). Indeed our current research on the cache protection strategies of scrub-jays stemmed from NSC's initial observations that western scrub-jays living on the University of California Davis campus readily took sandwiches and other food scraps left by humans and cached them in the surrounding grounds. Because several of the birds had color-rings, it was easy to discriminate individual birds. A jay would often cache food when other jays were nearby, and then fly off to a near by perch to wait until the other jays had left the scene. Then the cacher would return to recover these items and move them to new cache sites, a behavior we refer to as 're-caching'.

While field observations are essential for documenting natural behavior, an experimental approach is crucial for establishing whether these apparent cache protection strategies really can be attributed to the presence of other individuals. In a series of experiments in the laboratory, we therefore tested whether our jays would adjust their caching strategies to minimize potential stealing by other birds. To do so, the birds cached either in private (when the other bird's view was obscured) or while a conspecific was watching, and then recovered their caches in private 3 hours later. The procedure is illustrated in Figure 5. The point is that the cachers should only engage in cache protection strategies when another jay can observe the caching event; they should do not so when they cache in private. Furthermore, when it comes to recovery, if the purpose of re-caching is for cache protection then the birds should only bother to re-cache if they had been observed during the previous caching episode, but not if they had cached in private. Finally, the beauty of this design is that the conditions at recovery are identical and therefore the cacher's behavior at recovery cannot be attributed to any cues provided by the observer. Instead it must depend upon whether an observer was present during the previous caching episode, and, therefore, any difference in the behavior of the cacher at recovery must depend on its memory of the previous caching event (absence or presence of an observer jay). One final feature of the design is critical to the interpretation of these experiments, and that is that the birds received no more than 3 trials in each condition (observed and in-private) in any experiment. This is important because the birds were not given the opportunity to recover the caches that they had re-cached during recovery. Consequently, the birds had no opportunity to learn about the benefits of re-caching, an issue we shall return to at the end of this section.

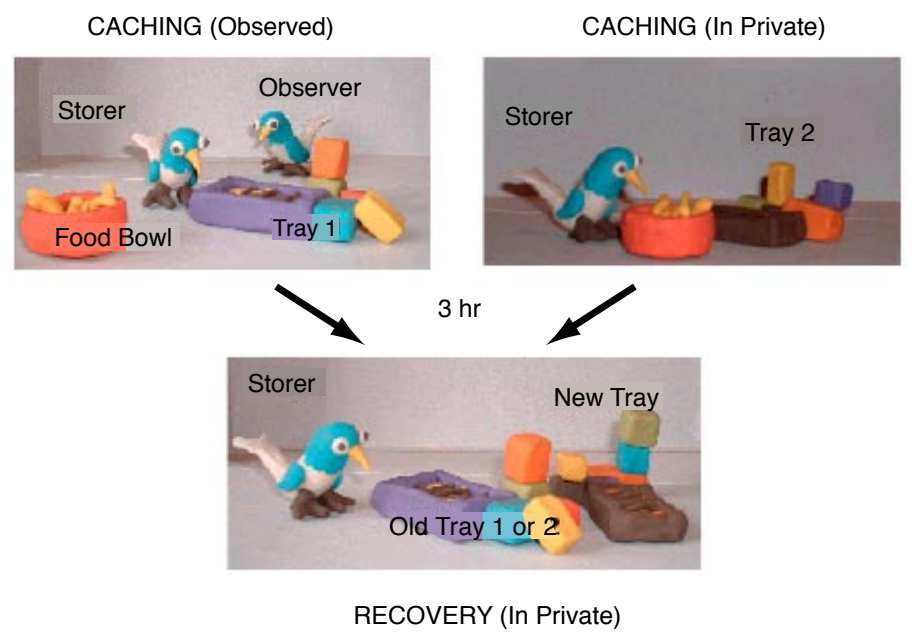

Figure 5. A cartoon of the procedure used to test whether the western scrub-jays could adjust their caching strategies to minimise potential stealing by other birds. On some trials the birds cached while observed by another jay (observed caching condition), whereas on other trials the birds cached in private (in-private caching condition). In all cases, the birds recovered their caches in private.

\section{Anticipating pilferage when observers are present}

We found that jays use a number of cache protection strategies at the time of caching, all of which appear to reduce the amount of visual information available to the observer. The first experiment examined the effects of sun and shade on a scrub-jay's choice of where to cache. We hypothesized that scrub-jays might prefer to cache in shady sites, especially when another bird was watching them, because shady sites would reduce the visual information available to the potential pilferer and thus reduce the chance that the pilferer could accurately relocate the caches. Figure 6 provides support for this hypothesis: When another jay is present at the time of 
caching, the cachers do prefer to hide most of their items in shady areas compared to well-lit areas, whereas they are not selective about cache location when caching in private (Dally, Emery, \& Clayton, 2004). Note, however, that not all the items were cached in the shady sites when an observer was watching: about $20 \%$ of the items were placed in the well-lit sites, a point we shall return to presently.

Subsequent experiments established that the jays also use distance and barriers as cache protection strategies. In the

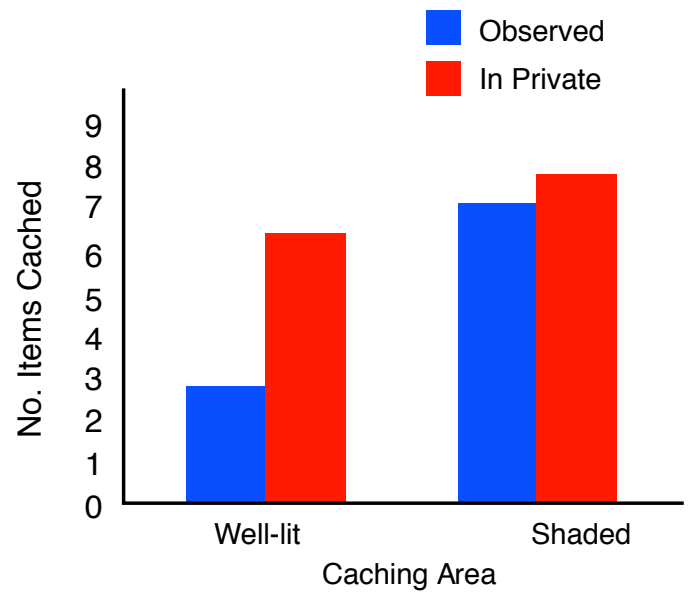

Figure 6. The mean number of items cached in well-lit ('sunny') and shaded cache sites during the observed and the in-private caching conditions of the sun-shade experiment of Dally et al (2004).

'distance' experiment, the birds were given the opportunity to cache in two trays, one near and one far from the observer's cage. The birds showed a preference to hide most of their caches in the tray located far from the observer, as opposed to the other tray placed near to the observer. However, they did not show a preference for distance when caching in private and instead cached equal amounts in both trays (Dally, Emery, \& Clayton, in press). In the 'barrier' experiment, the birds were given the opportunity to cache again in two trays, but now one was of them was placed behind a barrier but the other was in full view so that the observer could clearly see the location of caches hidden in the full-view tray, whereas those placed behind the barrier were out of its view. Perhaps not surprisingly, in the observed caching condition the jays cached preferentially in sites located behind barriers which observers could not see, whereas there was no preference to cache behind barriers when they cached in private. Note again that in both cases, when an observer was present during caching, the cacher placed about $25 \%$ of its caches in high-risk cache sites, namely in the near tray in the distance experiment, and in full-view tray in the barrier experiment.

\section{Cache protection at recovery when observers have left the scene}

As predicted, when the jays have been observed by another jay during caching then they also used another cache protection strategy - in the form of re-caching - during the recovery in private. By contrast, they engaged in much less re-caching when they had cached in private. Figure 7 shows the results for the sun-shade experiment. Note that it is the items that had been placed in the well-lit tray during the observed caching condition that were moved to new sites, even though the cacher had placed only about $20 \%$ of its caches in that tray. The same pattern of results was found for the distance and barrier experiments: those caches that had been placed in risky sites, namely those hidden in the near tray and those hidden in full-view of the observer, were the ones that were-re-cached but almost exclusively when they had been cached in the observed caching condition. Dally, Emery, and Clayton (in press) suggested that this behavior may also be a productive cache protection strategy, as in all cases at recovery, the jays re-cached only those items that were at risk. Note that in all cases, the birds re-cached the items in new locations that the observer did not know about, and they did so only when they had been observed during caching, but not when they had cached in private.

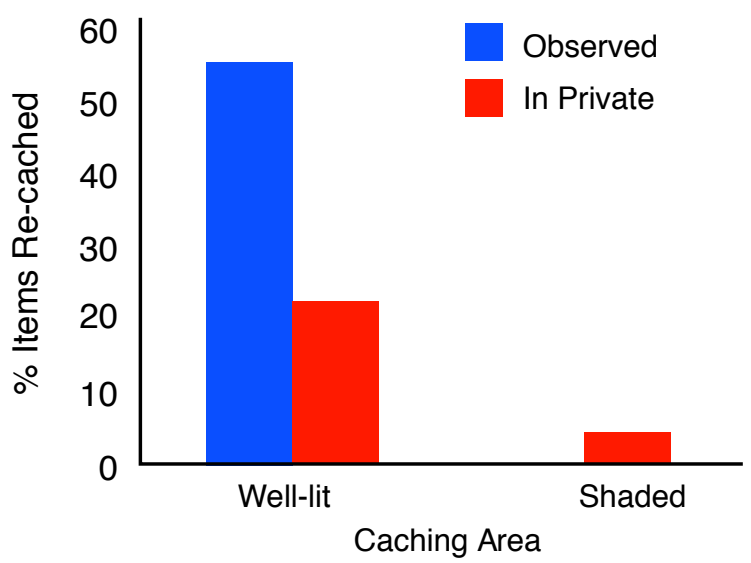

Figure 7. The number of items re-cached during caching as a percentage of the total number of items cached in the shaded and well-lit caching trays during recovery for both the observed and in-private caching conditions of the sunshade experiment of Dally et al, (2004).

There is one particularly striking finding about the recaching behavior of these birds, and that is that not all western scrub-jays engage in it. Emery and Clayton (2001) found that re-caching behavior depends not only on whether or not the cacher was observed by another jay during caching, but that it also depends upon experience of being a pilferer. Whereas experienced thieves engaged in high levels of re-caching at recovery when they were observed during the previous caching episode, control birds, who had not been thieves in the past and therefore had no prior experience of stealing other birds' caches, showed hardly any re-caching at all, as shown in Figure 8. 
That only experienced birds re-cache has a number of important implications. For example, re-caching cannot be innate, otherwise naïve and experienced scrub jays should both re-cache. We can also rule out a simple conditioning explanation because the birds in our experiments never received any positive reinforcement for re-caching. They never had the opportunity to recover the caches that they themselves had re-cached during these experiments. Instead, the inference is that the jays engaged in prospection. That is, they

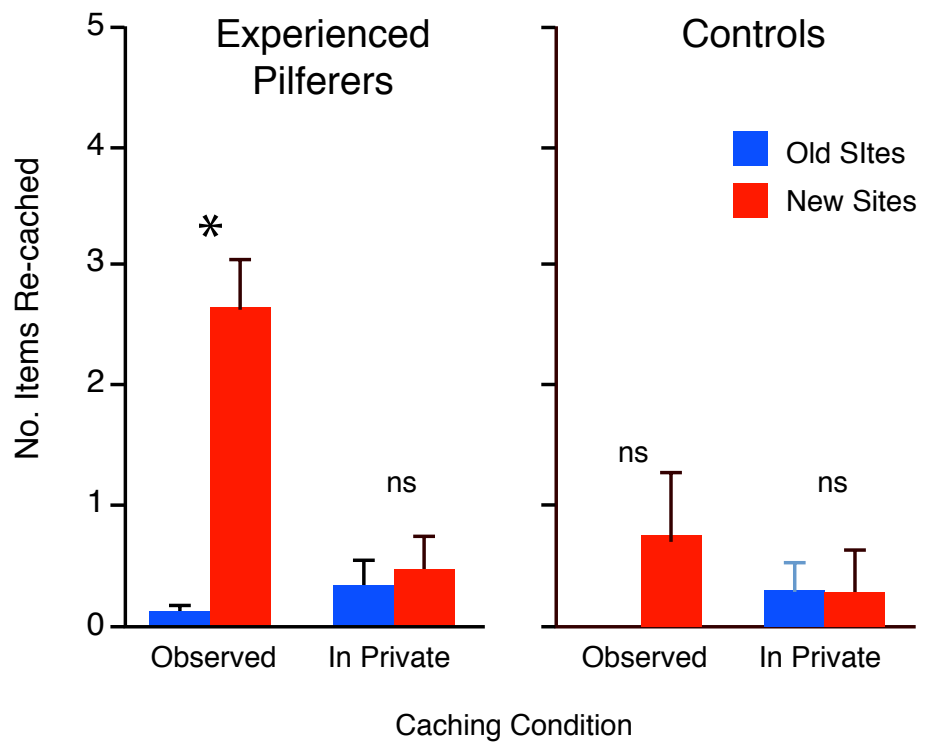

Figure 8. The mean number of items re-cached during the recovery period for both the observed and in-private caching conditions from Emery \& Clayton (2001). Caches were rarely moved to sites that were previously used during the caching period ('old sites'). Most of the caches are moved to 'new sites', which were the ones that were not used during the previous caching period and about which the observer bird has no information.

used information gained during previous caching events to anticipate whether or not their caches were likely to be stolen, and thus engaged in the appropriate cache protection strategy at recovery (whether to re-cache and, if so, to recache those caches that had previously been placed in highrisk sites). The fact that experienced birds differ so dramatically from control birds that lack the experience of being a thief suggests that the experienced jays are not only capable of prospection, but also capable of experience projection (Emery \& Clayton, 2004). Experience projection refers to one form of Theory of Mind (Premack \& Woodruff, 1978), namely the ability to use one's own experiences - in this case of having been a thief - to predict how another individual might think or behave - in this case what the potential pilferer might do. Experience projection has yet to be demonstrated in any of the great apes, other than in humans.

Our work has two, further important implications. The first is that elements of both prospective mental time travel and mental attribution appear to have evolved in, at least, two very disparate groups (apes and corvids), suggesting convergent evolution of these cognitive abilities (Emery \& Clayton, 2004). Second, the fact that corivids lack the typical six-layered cortex found in humans and some other mammals suggests that prospective cognition is achieved through different neurocognitive mechanisms in the avian and mammalian brain (Emery \& Clayton, in press).

\section{Conclusions:}

\section{The Mental Time Travel Hypothesis revisited.}

In summary, our studies have shown that scrub-jays are capable of short-term prospection in the control of searching for caches at recovery in way that can not be readily explained in terms of conditioning. More important for the mental time travel hypothesis, however, is the fact that caching itself appears to be cognitively prospective over a much longer period in that jays anticipate the state of caches at recovery and attempt to outwit the potential future behavior of conspecifics, Taken at face value, these findings challenge the exclusivity of human mental time travel, because the findings suggest that, when it comes to caching and protecting one's caches, at least one species of birds, the western scrub-jay, fulfils the criteria for long-term prospective cognition.

\section{References}

Adams, C. D., \& Dickinson, A. (1981). Instrumental responding following reinforcer devaluation. Quarterly Journal of Experimental Psychology, 33B, 109-122.

Baerends, G. P. (1941). Fortpflansungsverhalten und Orientierung der Grabwespe Ammophila campestris. Tidjscjrift voor Entomologie, 84, 68-275.

Berthold, P., Heilbig, A. J., Mohr, G. \& Querner, U. (1992). Rapid microevolution of migratory behaviour in a wild bird species. Nature, 360, 668-670. doi:10.1038/360668a0

Bugnyar, T. \& Kotrschal, K. (2002). Observational spatial learning and the raiding of food caches in ravens, Corvus corax: is it tactical deception. Animal Behaviour, 64, 185195. doi:10.1006/anbe.2002.3056

Clarkson, K., Eden, S. F., Sutherland, W. J. \& Houston, A. I. (1986). Density dependence and magpie food hoarding. Journal of Animal Ecology, 55, 111-121. doi: $10.2307 / 4696$

Clayton, N. S. \& Dickinson, A. (1999a). Motivational control of food storing in the Scrub jay, Aphelocoma coerulescens. Animal Behaviour, 57, 435-444.

doi:10.1006/anbe.1998.0989

Clayton, N. S. \& Dickinson, A. (1999b). Memory for the contents of caches by Scrub Jays. Journal of Experimental Psychology: Animal Behavior Processes, 25, 82-91. doi:10.1037/0097-7403.25.1.82

Clayton, N. S., Dally, J. M., Gilbert, J. D. J. \& Dickinson, A. 
(2005). Food Caching by Western Scrub-Jays (Aphelocoma californica) is sensitive to the conditions at recovery. Journal of Experimental Psychology: Animal Behavior Processes, 31, 115-124. doi:10.1037/0097-7403.31.2.115

Cook, R. G., Brown, M. F., \& Riley, D. L. (1985). Flexible memory processing by rats: Use of prospective and retrospective information in the radial maze. Journal of Experimental Psychology: Animal Behavior Processes, 11, 453-469. doi:10.1037/0097-7403.11.3.453

Curry, R. L., Peterson, T. \& Langen, T. A. (2002). Western Scrub-Jay. In: The Birds of North America 712, pp. 1-35, Poole, A. \& Gill, F. (Eds).

Dally, J. M., Emery, N. J. \& Clayton, N. S. (2004). Cache protection strategies by western scrub-jays (Aphelocoma californica): hiding food in the shade. Proceedings of the Royal Society: B Biological Letters, 271, 5387-5390.

Dally, J. M., Clayton, N. S. \& Emery, N. J. (In press). Cache protection strategies: behaviour, evolution and cognition. Animal Behaviour.

Dally, J. M., Emery, N. J. \& Clayton, N. S. (In press). Cache protection strategies by western scrub-jays: implications for social cognition. Animal Behaviour.

de Kort, S. D., Dickinson, A. \& Clayton, N. S. (2005). Retrospective cognition by food-caching western scrub-jays. Learning \& Motivation, 36, 159-176. doi:10.1016/j.Imot.2005.02.008

Emery, N. J \& Clayton, N. S. (2001). Effects of experience and social context on prospective caching strategies in scrub jays. Nature, 414, 443-446. doi:10.1038/35106560

Emery, N. J. \& Clayton, N. S. (2004). The mentality of crows. Convergent evolution of intelligence in corvids and apes. Science, 306, 1903-1907. doi:10.1126/science.1098410

Emery, N. J. \& Clayton, N. S. (In press). Evolution of avian brain and intelligence. Current Biology.

Fabre, J. H. (1916). The Hunting Wasps. Hodder and Stoughton: London.

Goodwin, D. 1955. Jays and crows recovering hidden food. British Birds, 48, 181-183.

Hall, G. (1996). Learning about associatively activated stimulus representations: Implications for acquired equivalence and perceptual learning. Animal Learning and Behavior, 24, 233-255.

Hampton, R. R., \& Sherry, D. F. (1992). The effects of cache loss on choice of cache sites in black-capped chickadees. Behavioral Ecology, 5, 44-50. doi:10.1093/beheco/5.1.44

Holland, P. C. (1990). Event representations in Pavlovian conditioning: Image and action. Cognition, 37, 105-131. doi:10.1016/0010-0277(90)90020-K

Honig, W. K., \& Thompson, R. K. R. (1982). Retrospective and propective processing in animal working memory. The Psychology of Learning and Motivation, 16, 239-283. doi:10.1016/S0079-7421(08)60551-4

Kamil, A. C., Balda, R. P., Olson, D. J., \& Good, S. (1993).
Returns to emptied cache sites by Clark's nutcrackers, $\mathrm{Nu}$ cifraga columbiana: a puzzle revisited. Animal Behaviour, 45, 241-252. doi:10.1006/anbe.1993.1030

McKenzie, T. L. B., Bird, L. R., \& Roberts, W. A. (2005). The effects of cache modification on food caching and retrieval behavior by rats. Learning and Motivation, 36, 260-278. doi:10.1016/j.lmot.2005.02.011

Premack, D. \& Woodruff, G. (1978). Does the chimpanzee have a theory of mind? Behavioral Brain Sciences, 1, 515526.

Roberts, W. A. (2002). Are animals stuck in time? Psychological Bulletin, 128, 473-489. doi:10.1037/0033-2909.128.3.473

Russell, J., \& Thompson, D. (2003). Memory development in the second year: for events or locations. Cognition, 87, B97-B105. doi:10.1016/S0010-0277(02)00238-X

Suddendorf, T. \& Corbalis, M. C. (1997). Mental time travel and the evolution of the human mind. Genetic and Social General Psychology Monographs, 123, 133-167.

Tulving, E. (1983). Elements of Episodic Memory Clarendon Press: Oxford.

Tulving, E. (2005). Episodic memory and Autonoesis: Uniquely Human? In H. Terrace \& J. Metcalfe (Eds), The Missing Link in Cognition. Origins in Self-Reflective Consciousness (pp. 3-56). Oxford University Press: Oxford.

Vander Wall, S. B. (1990). Food Hoarding in Animals. Chicago, University of Chicago Press.

Zentall, T. R. (1998). Symbolic representations in animals: Emergent stimulus relations in conditional discrimination learning. Animal Learning and Behavior, 26, 363-377. 